\title{
Steroids and osteoporosis: the quest for mechanisms
}

\author{
Stavros C. Manolagas
}

Division of Endocrinology and Metabolism, Center for Osteoporosis and Metabolic Bone Diseases, University of Arkansas for Medical Sciences and Central Arkansas Veterans Healthcare System, Little Rock, Arkansas, USA.

\begin{abstract}
Advances made during the last 35 years have improved our understanding of the mechanisms of steroid hormone action on bone and how physiologic, pathologic, or iatrogenic changes in hormone levels can lead to increased fracture risk. Estrogens, androgens, and glucocorticoids alter the cellular composition of bone by regulating the supply and lifespan of osteoclasts and osteoblasts. Additionally, they influence the survival of osteocytes, longlived cells that are entombed within the mineralized matrix and mediate the homeostatic adaptation of bone to mechanical forces. Altered redox balance is a proximal underlying mechanism of some of these effects, and sex steroid deficiency or glucocorticoid excess contributes to the aging of the skeleton.
\end{abstract}

Changes in steroid hormone levels are important factors in the development of osteoporosis, one of the most common metabolic disorders in the aging populations of the Western world. During the last 35 years, I had the privilege of partaking in the quest to elucidate the mechanisms of steroid hormone action on bone and the pathogenesis of osteoporosis. The following is a brief travelogue of some key discoveries that have shaped our thinking along the way.

Appreciation of the important role of gonads in the growth of the skeleton dates back to Aristotle (384-322 BCE), but it was Fuller Albright in the 1940s who first suggested a critical role of sex steroids in skeletal homeostasis during adulthood by pointing to an association between menopause and loss of bone mass (1). A decade earlier, Harvey Cushing had made the initial connection among the pituitary, the adrenals, and bone abnormalities (2). By the mid-1970s, when I started my thesis work on "Effect of Steroids on Bone," it had been well documented that decline or loss of gonadal function after adulthood increases the rate of bone turnover in animals and humans of either sex and leads to loss of bone mass and increased fracture risk. Moreover, iatrogenic glucocorticoid excess had become the second-most rec-

Conflict of interest: Stavros C. Manolagas serves on the scientific advisory board of Radius Health Inc. He has ownership of equity in this company and receives $\$ 10,000$ per annum for his SAB service.

Citation for this article: J Clin Invest. 2013; 123(5):1919-1921. doi:10.1172/JCI68062. ognizable cause of osteoporosis in women and men, both young and old.

The search for cellular and molecular mechanisms began in earnest with the detection in whole bone of high-affinity receptors for glucocorticoids and 1,25 dihydroxyvitamin $\mathrm{D}$, another steroid hormone with a profound role in mineral homeostasis (3). Identification of receptors for gonadal steroids in bone was more difficult, and it took another decade of intensive search before estrogen (and then androgen) receptors were detected in isolated bone cells $(4,5)$. The reason for the difficulty proved to be the much lower concentration of receptors for this class of steroid hormones in mature bone cells compared with reproductive organs. Unexpectedly, it was revealed 25 years later that estrogen receptor $\alpha(E R \alpha)$ in osteoblasts and osteoclasts mediates the protective effects of estrogen in cortical and cancellous bone, respectively. More surprisingly, ER $\alpha$ in osteoblasts stimulates cortical bone accrual in response to mechanical strain, independently of estrogens (6).

The detection of receptors for steroid hormones in bone cells automatically led to the idea that steroid hormones must modulate their biosynthetic activity. Not long after, it was shown that steroid hormones do not merely alter the function of existing bone cells, but are in fact potent regulators of their generation and lifespan. The linchpin for this conceptual advance was the discovery of 1,25 dihydroxyvitamin $D$ receptors in monocytes and activated $\mathrm{T}$ lymphocytes (7) and the effects of 1,25 dihydroxyvita- min D on monocyte differentiation and on cytokine production by T cells (8). The relevance of these findings to other steroid hormones soon became evident with the demonstration of potent effects of estrogens and androgens on osteoclastogenesis via cytokine-mediated mechanisms $(9,10)$.

In two papers published in JCI two months apart in 1998, we demonstrated that both estrogens and glucocorticoids restrain osteoblastogenesis and that glucocorticoids shorten the lifespan of mature osteoblasts and osteocytes by stimulating apoptosis $(11,12)$. Shortly after, it was found that estrogens and androgens prolong osteoblast and osteocyte survival by attenuating apoptosis $(13,14)$. On the other hand, estrogens and androgens shorten the lifespan of osteoclasts, whereas glucocorticoids prolong it (15). Bone cell apoptosis has since become an intense focus in the study of the pathogenesis of osteoporosis, as well as of the mechanism of action of anti-osteoporotic drugs (16). This body of work has clearly established that steroid hormones influence the cellular composition of bone as opposed to merely regulating existing cell function (17). Moreover, it directed us to think of osteocytes as important cellular targets of steroid hormone action. Parallel advances in osteocyte biology heightened the significance of this concept. It is now well appreciated that this third and most abundant bone cell type plays a central role in the choreography of bone remodeling by controlling rate-limiting steps of bone resorption and formation. Osteocytes are the major cellular source of RANKL for physiologic as well as pathologic bone remodeling (18), and mounting evidence suggests that osteocyte death by apoptosis and/or dysfunction, such as from compromised autophagy, plays a seminal role in the pathogenesis of involutional, postmenopausal, glucocorticoid- and immobilization-induced osteoporosis (19).

Elucidation of the effects of steroid hormones in the birth and death of bone 


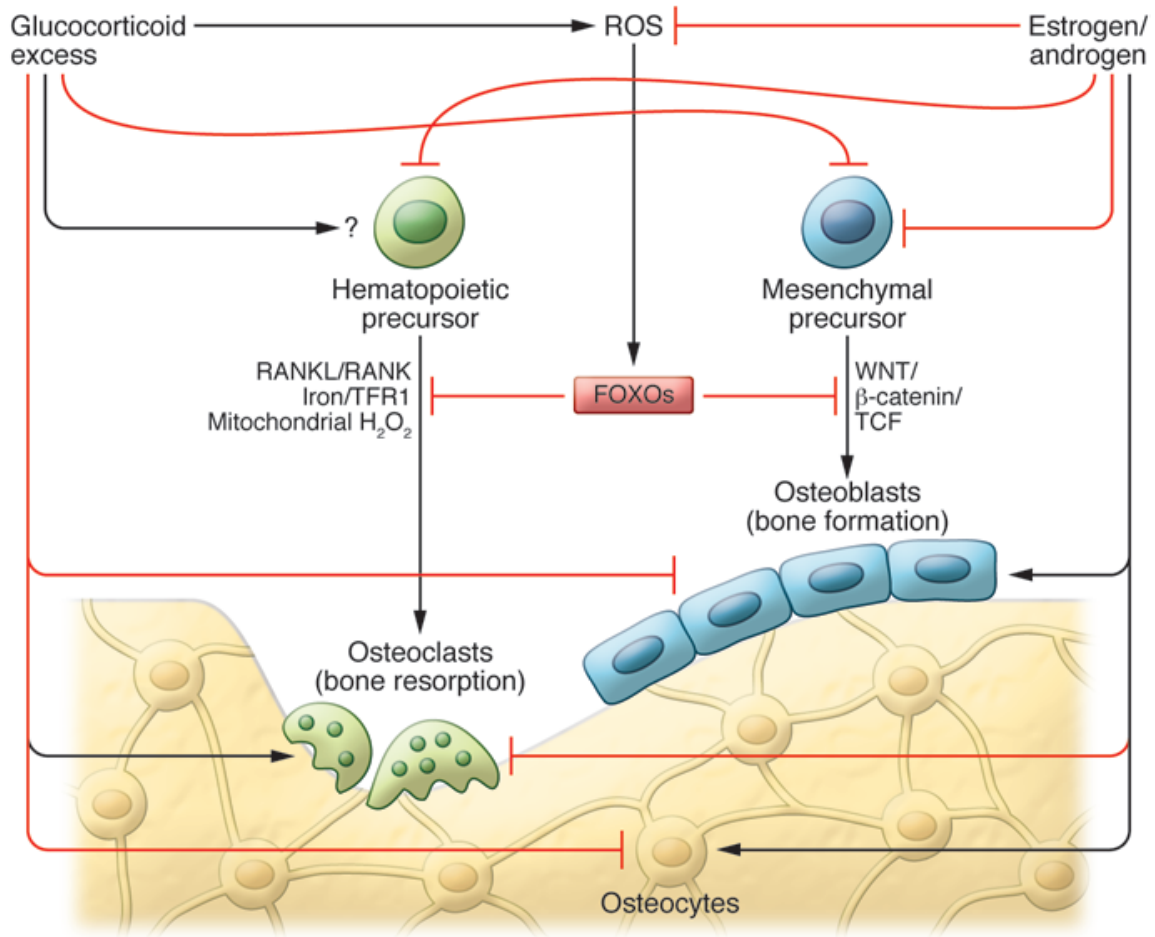

Figure 1

Steroid hormones influence the birth and death of bone cells. Osteoclasts and osteoblasts are derived from hematopoietic and mesenchymal precursors, respectively. Osteoclast generation, function, and survival require RANKL signaling via its receptor, RANK, as well as mitochondrial biogenesis coupled with iron uptake through transferrin receptor 1 (TFR1) and iron supply to mitochondrial respiratory proteins; both cascades lead to increased $\mathrm{H}_{2} \mathrm{O}_{2}$ generation, which promotes osteoclastogenesis. Osteoblast generation is critically dependent on WNT/ $\beta$-catenin/TCF signaling. ROS, which are primarily generated by the mitochondrial respiratory chain, activate FOXO transcription factors that suppress $\mathrm{H}_{2} \mathrm{O}_{2}$ production and decrease osteoclastogenesis. ROS also decrease the proliferation/differentiation of committed osteoblast precursors, by a diversion of $\beta$-catenin from WNT/ $\beta$-catenin/TCF- to FOXO-mediated transcription. During the process of bone remodeling, bone matrix excavated by osteoclasts is replaced with new matrix produced by osteoblasts. Glucocorticoids (at supraphysiologic levels) and estrogens or androgens influence the generation and lifespan of osteoclasts and osteoblasts and the lifespan of osteocytes, at least in part, by altering redox balance. Black and red lines pointing to mature osteoclasts and osteoblasts on the bone surface and osteocytes embedded in the matrix indicate prolongation and shortening effects, respectively, on lifespan.

cells (Figure 1) opened a new chapter of the quest: the exploration of their role in redox homeostasis. During the last ten years, studies in animal models have revealed that advancing age progressively increases oxidative stress in the skeleton (20). ROS attenuate osteoblastogenesis and shorten the lifespan of osteoblasts and osteocytes. On the other hand, ROS are required for osteoclast generation, function, and survival. Increased ROS generation leads to the activation of FOXOs, a family of transcription factors that represent an important defense mechanism against oxidative stress. In osteoblasts, as in other cell lineages, FOXOs provide an optimal balance among the maintenance of self-renewing stem cells, the replication of lineage-committed progenitors, and the survival of their terminally differentiated progeny. On the other hand, FOXOs inhibit osteoclast generation by attenuating ROS. Estrogens or androgens decrease oxidative stress $(20,21)$, whereas glucocorticoids enhance it (22). Conversely, estrogen or androgen deficiency increases ROS generation and $\mathrm{p} 53$ activation in a manner similar to aging. Moreover, the adverse effects of estrogen or androgen loss on bone in the murine model are prevented by antioxidants. Thus, age-related changes in the production of steroids in the ovaries and the adrenals, (23) as well as iatrogenic hyperglucocorticoidism, contribute to the pathogenesis of osteoporosis by accentuating age-related mechanisms intrinsic to bone and oxidative stress.

I expect that in years to come, understanding of the mechanisms I discussed here will evolve, additional mechanisms will be elucidated, and the target genes of steroid hormone action on bone will become much clearer than they are now. What I believe will endure, however, are the overarching themes that for regenerating tissues, such as bone, cell number is more important than cell vigor; that different steroid hormones affect very similar cellular processes; and that age-related mechanisms are protagonists in the pathogenesis of osteoporosis, and changes in steroid hormones are contributory. The satisfaction and joy of sharing this journey with a synergistic research team has been an added personal bonus for this traveler.

\section{Acknowledgments}

I thank Robert Jilka, Michael Parfitt, Robert Weinstein, Charles O’Brien, Maria Almeida, Teresita Bellido, Stavroula Kousteni, Haibo Zhao, and the rest of my current and former coworkers and trainees for their invaluable input over these many years; and Leah Elrod for help with the preparation of the manuscript.

Address correspondence to: Stavros C. Manolagas, 4301 West Markham, \#587, Little Rock, Arkansas 72205-7199, USA. Phone: 501.686.5130; Fax: 501.686.8148; E-mail: manolagasstavros@uams.edu.

1. Reifenstein EC, Albright F. The metabolic effects of steroid hormones in osteoporosis. J Clin Invest. 1947;26(1):24-56.

2. Cushing $H$. The basophil adenomas of the pituitary body and their clinical manifestations (pituitary basophilism). Bull Johns Hopkins Hosp. 1932; 50(5):137-195

3. Manolagas SC, Anderson DC, Lumb GA. Glucocorticoids regulate the concentration of 1,25-dihydroxycholecalciferol receptors in bone. Nature. 1979; 277(5694):314-315

4. Eriksen EF, et al. Evidence of estrogen receptors in normal human osteoblast-like cells. Science. 1988; 241(4861):84-86.

5. Komm BS, et al. Estrogen binding, receptor mRNA, and biologic response in osteoblast-like osteosarcoma cells. Science. 1988;241(4861):81-84.

6. Almeida M, et al. Estrogen receptor-alpha signaling in osteoblast progenitors stimulates cortical bone accrual. J Clin Invest. 2013;123(1):394-404.

7. Provvedini DM, Tsoukas CD, Deftos LJ, Manolagas SC. 1,25-Dihydroxymitamin $\mathrm{D}_{3}$ receptors in human leukocytes. Science. 1983;221(4616):1181-1183.

8. Tsoukas CD, Provvedini DM, Manolagas SC. 1,25-Dihydroxyvitamin $\mathrm{D}_{3}$ : a novel immunoregulatory hormone. Science. 1984;224(4656):1438-1440.

9. Jilka RL, et al. Increased osteoclast development after estrogen loss: Mediation by interleukin-6. Science. 1992;257(5066):88-91. 
10. Bellido T, et al. Regulation of interleukin-6, osteoclastogenesis and bone mass by androgens: the role of the androgen receptor. J Clin Invest. 1995; 95(6):2886-2895.

11. Jilka RL, Takahashi K, Munshi M, Williams DC, Roberson PK, Manolagas SC. Loss of estrogen upregulates osteoblastogenesis in the murine bone marrow: evidence for autonomy from factors released during bone resorption. J Clin Invest. 1998;101(9):1942-1950.

12. Weinstein RS, Jilka RL, Parfitt AM, Manolagas SC. Inhibition of osteoblastogenesis and promotion of apoptosis of osteoblasts and osteocytes by glucocorticoids: potential mechanisms of their deleterious effects on bone. J Clin Invest. 1998;102(2):274-282.

13. Kousteni S, et al. Nongenotropic, sex-nonspecific signaling through the estrogen or androgen receptors: dissociation from transcriptional activity. Cell. 2001;104(5):719-730.

14. Kousteni S, et al. Reversal of bone loss in mice by nongenotropic signaling of sex steroids. Science.
2002;298(5594):843-846.

15. Weinstein RS, et al. Promotion of osteoclast survival and antagonism of bisphosphonate-induced osteoclast apoptosis by glucocorticoids. J Clin Invest. 2002;109(8):1041-1048.

16. Jilka RL, Weinstein RS, Bellido T, Roberson P, Parfitt AM, Manolagas SC. Increased bone formation by prevention of osteoblast apoptosis with parathyroid hormone. J Clin Invest. 1999;104(4):439-446.

17. Manolagas SC. Birth and death of bone cells: basic regulatory mechanisms and implications for the pathogenesis and treatment of osteoporosis. Endocr Rev. 2000;21(2):115-137.

18. Xiong J, Onal M, Jilka RL, Weinstein RS, Manolagas SC, O’Brien CA. Matrix-embedded cells control osteoclast formation. Nature Medicine. 2011; 17(10):1235-1241.

19. Manolagas SC, Parfitt AM. For whom the bell tolls: Distress signals from long-lived osteocytes and the pathogenesis of metabolic bone diseases. Bone.
2012;pii:S8756-3282(12)01246-X.

20. Manolagas SC. From estrogen-centric to aging and oxidative stress: a revised perspective of the pathogenesis of osteoporosis. Endocr Rev. 2010; 31(3):266-300.

21. Almeida M, Han L, Ambrogini E, Bartell SM, Manolagas SC. Oxidative stress stimulates apoptosis and activates NF-kappaB in osteoblastic cells via a PKCbeta/p66shc signaling cascade: counter regulation by estrogens or androgens. Mol Endocrinol. 2010;24(10):2030-2037.

22. Almeida M, Han L, Ambrogini E, Weinstein RS, Manolagas SC. Glucocorticoids and tumor necrosis factor (TNF) alpha increase oxidative stress and suppress WNT signaling in osteoblasts. J Biol Chem. 2011;286(52):44326-44335.

23. Weinstein RS, et al. Endogenous glucocorticoids decrease skeletal angiogenesis, vascularity, hydration, and strength in aged mice. Aging Cell. 2010; 9(2):147-161. 